\title{
Computation of the Strand Resistance Using the Core Wire Strain Measurement
}

\author{
Keunhee Cho, Sung Tae Kim, Sung Yong Park, Young-Hwan Park* \\ Structural System Research Division, Korea Institute of Construction Technology, Goyang-Si, Republic of Korea \\ Email: kcho@kict.re.kr, esper009@kict.re.kr, sypark@kict.re.kr, yhpark@kict.re.kr
}

Received August 5, 2013; revised September 5, 2013; accepted September 12, 2013

Copyright (C) 2013 Keunhee Cho et al. This is an open access article distributed under the Creative Commons Attribution License, which permits unrestricted use, distribution, and reproduction in any medium, provided the original work is properly cited.

\begin{abstract}
This paper proposes a method enabling to compute the prestressing strand resistance using the strain measured on only one core wire. Numerical analysis is conducted considering the pitch length of the strand and the diameters of the core wire and helical wires as parameters. The results verify that the relation between the stresses of the core wire and helical wires can be expressed in terms of the helical angle. Based on this observation, a formula computing directly the prestress force in the strand from the strain measured in the core wire is suggested. Owing to the recently developed measurement method for the core wire strain, the proposed formula can be exploited to determine the prestress of the strand.
\end{abstract}

Keywords: Finite Element Method; Measurement; Prestress; Strand; Core Wire; Helical Wire

\section{Introduction}

Prestress is introduced in concrete structures to reduce the quantity of materials and achieve longer span. The evaluation of the changes in the prestress according to the level of prestress and use of the structure is of utmost importance to assess the state of the structure. This prestress is generally introduced by means of strands. Accordingly, the resistance of the strand should be measured continuously along the service life to evaluate the level of prestress.

Among the methods that have been developed to measure the prestress of the strand, methods using guided stress wave [1,2], elastomagnetic stress sensor [3], and fiber optic sensor [4] are currently applied. Recently, a method using fiber optic sensor and measuring the strain of one wire instead of the whole strand has been developed [5]. This method differs from previous methods, which measure the resistance of the whole strand, by measuring the strain of only one wire constituting the strand. Therefore, need is for a method estimating the resistance of the whole strand from the strain measured on one wire.

A strand is composed of a straight core wire surrounded by helical wires. The difference in the shapes of the core wire and helical wires provokes the development

\footnotetext{
*Corresponding author.
}

of different stresses. Analytic studies [6,7] and numerical studies [8-10] have been carried out to identify the behavior of the strand and its load-resisting characteristics. However, these studies focused on the behaviors of the whole strand or individual core wire or individual helical wire without providing any method estimating the resistance of the whole strand based on the strain of an individual wire.

Accordingly, this study intends to propose a method enabling to compute the prestress force of the strand by measuring the strain of one wire. First, numerical analysis is conducted for 7-wire strands with diameter of 15.2 $\mathrm{mm}$ and presenting diverse shapes. The results are used to determine rational measurement positions on the wires and to derive the relationship between the stresses of the wires. Finally, a relation between the measured strain and the resistance of the strand is suggested.

\section{Selection of Analysis Parameters and Material Properties}

Parametric study is conducted to examine the behavioral pattern of the strand according to the change of its shape. The shape of the strand is determined with respect to the diameter $D_{c}$ of the core wire, the diameter $D_{h}$ of the helical wires, and the pitch length $L_{p}$ (Figure 1). In addition, the shape can also be expressed in terms of the helical 

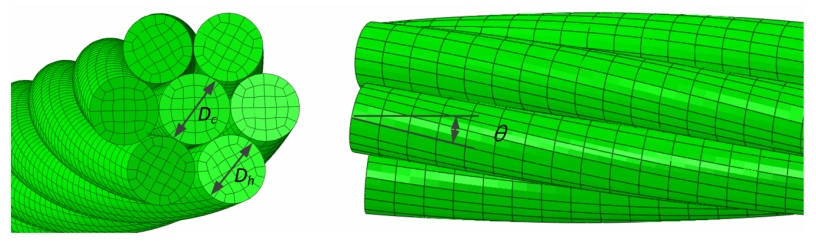

Figure 1. Shape and element meshing of the 7-wire strand model.

angle $(\theta)$ between the axis of the core wire and the axis of the helical wire. In general, the diameter of the core wire is larger than that of the helical wire in order to reduce the frictional effect between the helical wires. The KS standards (2011) [11] specify that, for a 7-wire strand, the diameter of the core wire shall be larger by minimum $0.08 \mathrm{~mm}$ than that of the helical wire and that the pitch length shall be 12 to 18 times larger than the diameter of the 7-wire strand $(D)$.

Accordingly, the difference in the diameters of the core wire and helical wire and the pitch length can be selected as variables for the shape of the 7-wire strand. Therefore, the diameter of the 7-wire strand is fixed to $15.2 \mathrm{~mm}$ and the difference between the diameters of the core wire and helical wire is set to range from $0.08 \mathrm{~mm}$ to $0.20 \mathrm{~mm}$ by step of $0.04 \mathrm{~mm}$ (Table 1). The pitch length is varied from 12 times to 18 times the diameter of the 7-wire strand by unit step (Table 2). The analysis is performed for 28 cases involving 4 cases with different diameters between the core wire and helical wire and 7 different pitch lengths.

The core wire and helical wires of the 7-wire strand are modeled using three-dimensional 20-node continuum elements (Figure 1). The interaction between the core wire and helical wires is represented by means of contact and a coefficient of friction of 0.115 is applied [12]. Since the strand is actually tensioned to a level lower than the yield strength, the material is modeled to be linearly elastic. The elastic modulus is $200 \mathrm{GPa}$ and the Poisson's ratio is 0.3 .

The end sections of the strand are modeled as rigid bodies. In order to prevent any effect of such end constraints on the analysis results, the length of the strand is extended sufficiently to be twice the pitch length. All the degrees-of-freedom on one end are restrained and the degrees-of-freedom at the other end are restrained at the exception of the degree-of-freedom in the longitudinal direction. A load of $200 \mathrm{kN}$ is applied in the longitudinal direction.

\section{Analysis Results}

Contact between the helical wires occurred under the application of the load for some cases where the difference between the diameters of the core wire and helical wire was small. However, since the strand is fabricated to
Table 1. Parameter related to the difference between the diameters of the core wire and helical wire (for $15.2 \mathrm{~mm}$ diameter strand).

\begin{tabular}{ccccc}
\hline$D_{c}-D_{h}(\mathrm{~mm})$ & 0.08 & 0.12 & 0.16 & 0.20 \\
\hline$D_{c}(\mathrm{~mm})$ & 5.12 & 5.15 & 5.17 & 5.20 \\
$D_{h}(\mathrm{~mm})$ & 5.04 & 5.03 & 5.01 & 5.00 \\
\hline
\end{tabular}

Table 2. Pitch length parameter as multiple of the diameter of the 7-wire strand (for $\mathbf{1 5 . 2} \mathbf{~ m m}$-diameter strand).

\begin{tabular}{cccccccc}
\hline $\begin{array}{c}\text { Pitch length } \\
\text { multiple }\end{array}$ & 12 & 13 & 14 & 15 & 16 & 17 & 18 \\
\hline $\mathrm{L}_{\mathrm{p}}(\mathrm{mm})$ & 182.4 & 197.6 & 212.8 & 228.0 & 243.2 & 258.4 & 273.6 \\
\hline
\end{tabular}

prevent practically any contact between the helical wires, the cases in which contact between the helical wires occurred were discarded from the analysis (Table 3).

In order to compute the resistance of the strand based upon the strain measured on the core wire, this measured strain should be representative of the strain of the core wire. If the resistance of the strand is computed using the strain measured locally at a spot where strain concentration occurs due to contact or any other reason, erroneous overestimation of the prestress would be achieved. Therefore, the longitudinal distribution of the stress in the section at mid-length of the strand is drawn to verify the appropriate position on the core wire at which strain should be measured (Figure 2). The stress in the core wire exhibits nearly uniform distribution at the exception of its circumferential contour in contact with the helical wires. The stress in the helical wires appears to be practically constant with regard to the whole section in case of small difference between the diameters but tends to reduce as much as the distance from the center of the strand increases in case of large difference between the diameters.

To verify the consistency of this sectional stress distribution over the whole length of the strand, the longitudinal stress of the core wire is plotted for the whole length of the strand (Figure 3). Here, the normalized distance corresponds to the longitudinal position on the strand divided by the length of the strand. At the exception of some parts at the extremities, the stress at the central axes of the core wire and helical wire are seen to remain constant. Besides, the stress at the edge of the core wire varies continuously due to the contact with the helical wires. This stress varies in a range from $96.5 \%$ to $102.1 \%$ of the stress at the center axis of the core wire. Accordingly, the measurement of the stress of the core wire at the edge would result in an error of $-3.5 \%$ to $2.1 \%$.

\section{Discussion}

The objective of this study is to compute the resistance of 

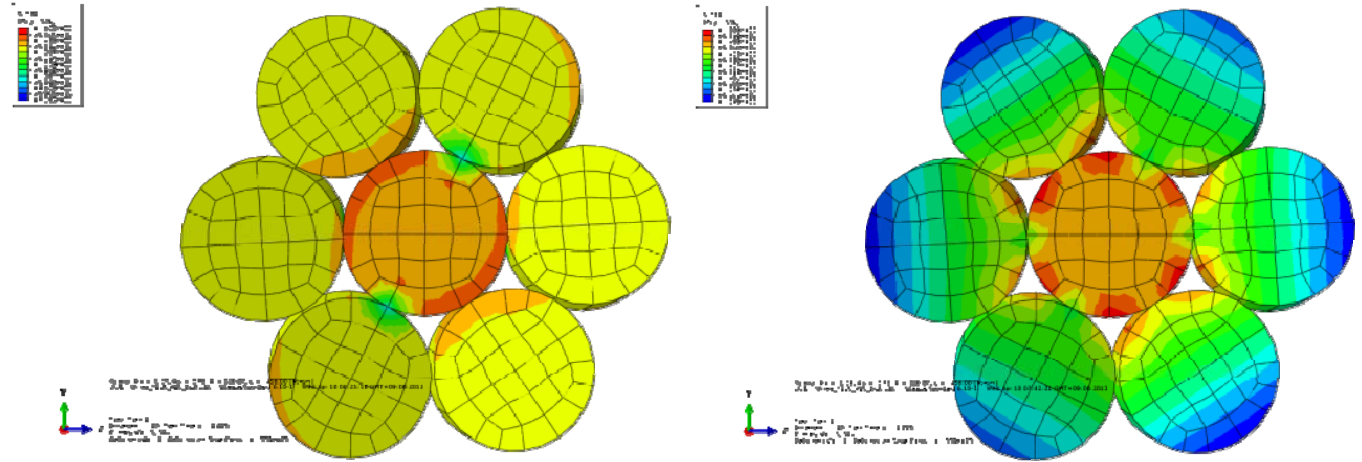

(a)

(b)
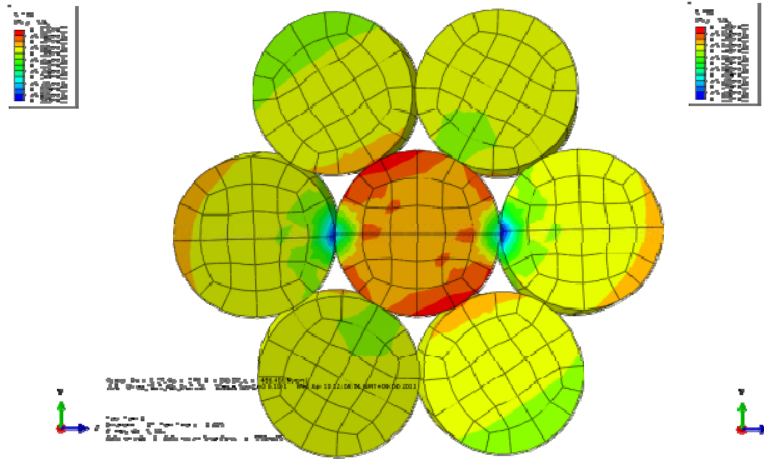

(c)

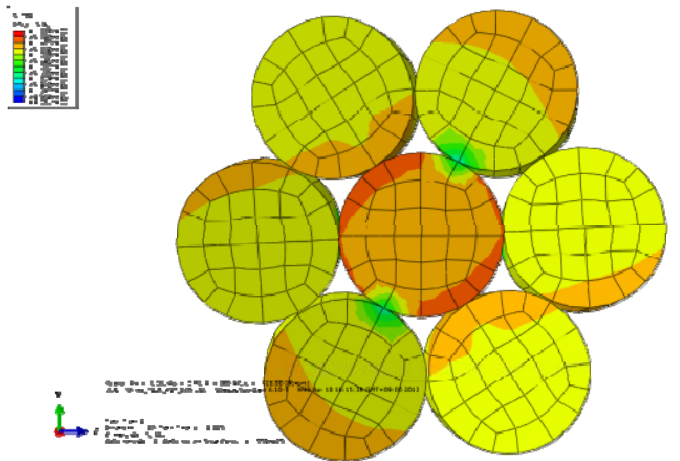

(e)

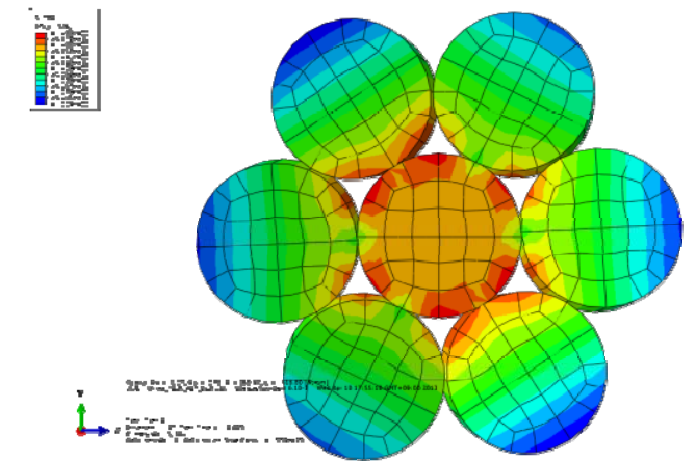

(f)

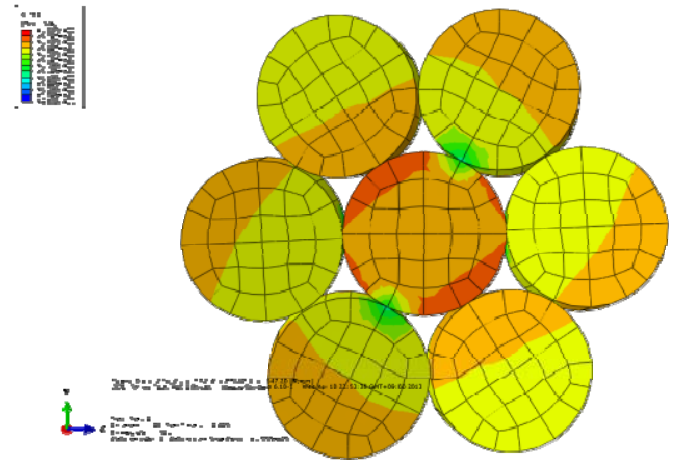

(g)

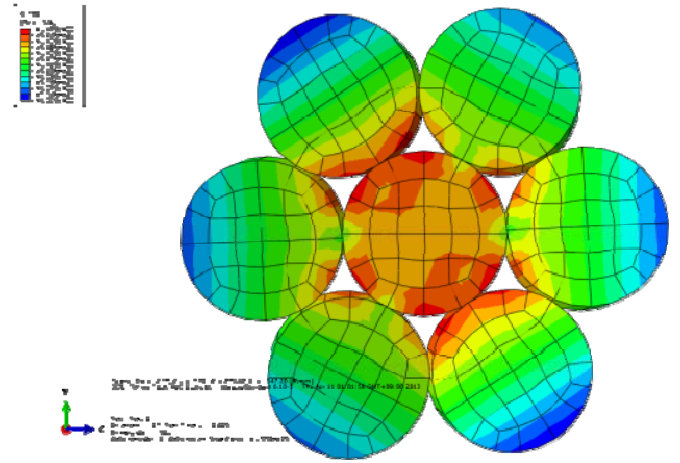

(h)

Figure 2. Longitudinal stress distribution at mid-length section of the strand. (a) Pitch length $=15, D_{c}-D_{h}=0.12$ mm; (b) Pitch length $=15, D_{c}-D_{h}=0.16 \mathrm{~mm} ;$ (c) Pitch length $=16, D_{c}-D_{h}=0.12 \mathrm{~mm} ;$ (d) Pitch length $=16, D_{c}-D_{h}=0.16 \mathrm{~mm} ;($ e) Pitch length $=17, D_{c}-D_{h}=0.08 \mathrm{~mm}$; (f) Pitch length $=17, D_{c}-D_{h}=0.12 \mathrm{~mm} ;\left(\right.$ g) Pitch length $=18, D_{c}-D_{h}=0.08 \mathrm{~mm} ;(\mathrm{h})$ Pitch length $=18, D_{c}-D_{h}=0.12 \mathrm{~mm}$. 


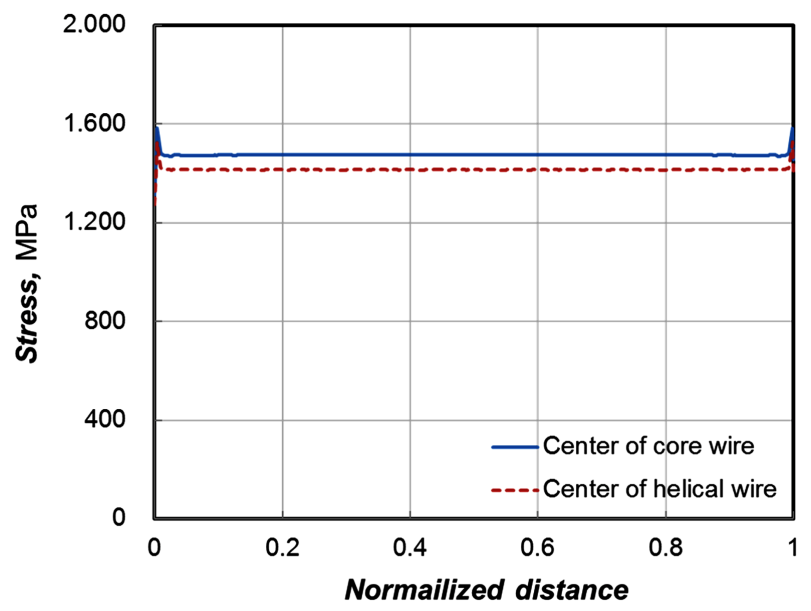

(a)

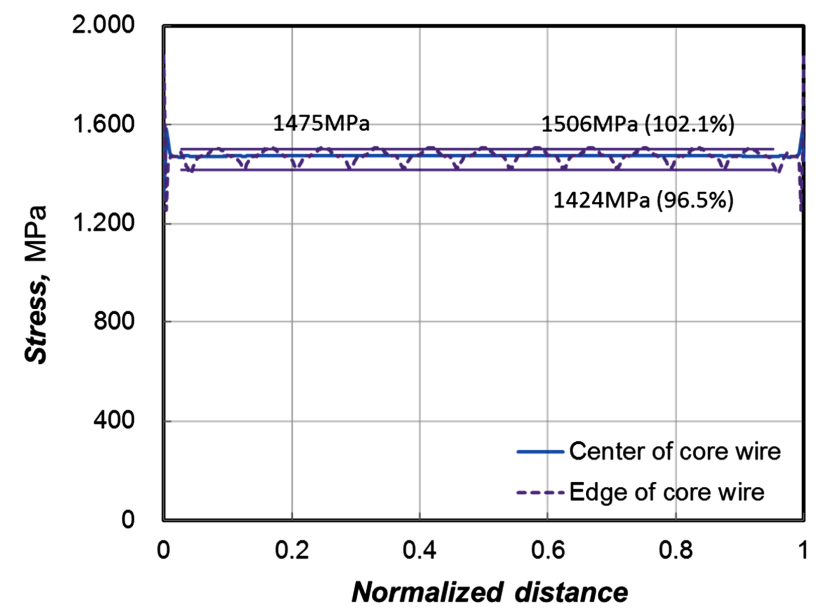

(b)

Figure 3. Longitudinal stress distribution in the core wire and helical wire for the whole length of the strand (pitch length $=$ $15 D, D_{c}-D_{h}=0.16 \mathrm{~mm}$ ). (a) Stresses at centers of core wire and helical wire; (b) Stresses at center and edge of core wire.

Table 3. Analysis cases excluding the cases in which contact between the helical wires occurred.

\begin{tabular}{cc}
\hline$D_{c}-D_{h}(\mathrm{~mm})$ & Pitch length multiple \\
\hline 0.08 & $12,13,14,15$ \\
0.12 & 12 \\
\hline
\end{tabular}

the strand by measuring the strain of the core wire. To that goal, the mean strain of the core wire should be measured or derived from the measurements and, the corresponding stress of the helical wire should be estimated from the measured strain of the core wire. Since, apart from its edge, the core wire exhibits nearly constant stress state, the mean strain can be obtained by measureing the strain at the center of the core wire. Besides, the mean stress of the helical wire cannot be obtained directly by analysis because the stress distribution shows different patterns according to the difference in the diameters.

This study intends to obtain the mean stress of the helical wire as follows. First, the mean stress $\left(\sigma_{c}\right)$ and resistance $\left(F_{c}\right)$ of the core wire are calculated using the strain $\left(\varepsilon_{c}\right)$ measured at the center of the core wire.

$$
\begin{aligned}
& \sigma_{c}=E_{s} \varepsilon_{c} \\
& F_{c}=A_{c} \sigma_{c}
\end{aligned}
$$

where $E_{s}$ is the Young's modulus and, $A_{c}$ is the cross-sectional area of the core wire. Thereafter, the resistance $\left(F_{h}\right)$ of the helical wire is obtained by subtracting the force $(F)$ applied on the strand from the so-obtained resistance of the core wire.

$$
F_{h}=F-F_{c}
$$

Finally, the mean stress $\left(\sigma_{h}\right)$ of the helical wire is obtained by dividing the resistance of the helical wire by its cross-sectional area $\left(A_{h}\right)$.

$$
\sigma_{h}=F_{h} / A_{h}
$$

The ratio of the stress of the helical wire to the stress of the core wire per shape of the strand computed through this process ranges between 0.944 and 0.977 (Table 4). It can be seen that the stress of the helical wire converges to the stress of the core wire as much as the pitch length becomes longer, and that the difference between the diameters of the core and helical wires does not have noticeable effect of the stress ratio.

The relation between the stresses of the core wire and helical wire is formulated as function of the helical angle $(\theta)$, itself related to the pitch length and difference between the diameters. The helical angle is the angle described by the longitudinal axis of the core wire and longitudinal axis of the helical wire, and can be obtained by Equation (5).

$$
\tan \theta=\frac{D_{c}+D_{h}}{2 L_{p}}
$$

Figure 4 plotting the stress ratio according to the helical angle is in good agreement with the analytic solution (Costello, 1997). The stress ratio decreases gradually with larger helical angle and is practically linearly proportional to $\tan \theta$. Accordingly, the relation between the helical angle and stress ratio can be obtained through linear regression analysis and is expressed in Equation (6). This formula enables thus to estimate the stress of the helical wire from the stress of the core wire by using only the shape of the strand.

$$
\frac{\sigma_{h}}{\sigma_{c}}=-0.5214 \tan \tan \theta+1.0388
$$

Finally, the prestress of the strand can be obtained as 
Table 4. Mean stress and resistance of core wire and helical wire per shape of strand.

\begin{tabular}{|c|c|c|c|c|c|c|c|}
\hline Pitch length multiple & Diameter difference (mm) & $\tan \theta$ & $\sigma_{c}(\mathrm{MPa})$ & $F_{c}(\mathrm{kN})$ & $F_{h} \quad(\mathrm{kN})$ & $\sigma_{h} \quad(\mathrm{MPa})$ & $\frac{\sigma_{h}}{\sigma_{c}}$ \\
\hline 12 & 0.16 & 0.1755 & 1505.2 & 31.6 & 168.4 & 1421.5 & 0.944 \\
\hline 12 & 0.20 & 0.1757 & 1504.5 & 32.0 & 168.0 & 1426.5 & 0.948 \\
\hline 13 & 0.12 & 0.1617 & 1490.0 & 31.0 & 169.0 & 1419.4 & 0.953 \\
\hline 13 & 0.16 & 0.1620 & 1493.9 & 31.4 & 168.6 & 1423.5 & 0.953 \\
\hline 13 & 0.20 & 0.1622 & 1494.1 & 31.7 & 168.3 & 1428.3 & 0.956 \\
\hline 14 & 0.12 & 0.1502 & 1480.2 & 30.8 & 169.2 & 1421.1 & 0.960 \\
\hline 14 & 0.16 & 0.1504 & 1481.5 & 31.1 & 168.9 & 1425.7 & 0.962 \\
\hline 14 & 0.20 & 0.1506 & 1486.0 & 31.6 & 168.4 & 1429.8 & 0.962 \\
\hline 15 & 0.12 & 0.1402 & 1473.2 & 30.6 & 169.4 & 1422.3 & 0.965 \\
\hline 15 & 0.16 & 0.1404 & 1475.1 & 31.0 & 169.0 & 1426.8 & 0.967 \\
\hline 15 & 0.20 & 0.1405 & 1479.6 & 31.4 & 168.6 & 1430.9 & 0.967 \\
\hline 16 & 0.08 & 0.1312 & 1463.7 & 30.1 & 169.9 & 1419.1 & 0.969 \\
\hline 16 & 0.12 & 0.1314 & 1467.8 & 30.5 & 169.5 & 1423.2 & 0.970 \\
\hline 16 & 0.16 & 0.1316 & 1470.0 & 30.9 & 169.1 & 1427.7 & 0.971 \\
\hline 16 & 0.20 & 0.1318 & 1474.4 & 31.3 & 168.7 & 1431.9 & 0.971 \\
\hline 17 & 0.08 & 0.1235 & 1458.8 & 30.0 & 170.0 & 1419.9 & 0.973 \\
\hline 17 & 0.12 & 0.1237 & 1461.3 & 30.4 & 169.6 & 1424.4 & 0.975 \\
\hline 17 & 0.16 & 0.1238 & 1465.8 & 30.8 & 169.2 & 1428.5 & 0.975 \\
\hline 17 & 0.20 & 0.1240 & 1470.2 & 31.2 & 168.8 & 1432.6 & 0.974 \\
\hline 18 & 0.08 & 0.1167 & 1454.9 & 30.0 & 170.0 & 1420.6 & 0.976 \\
\hline 18 & 0.12 & 0.1168 & 1457.9 & 30.3 & 169.7 & 1425.0 & 0.977 \\
\hline 18 & 0.16 & 0.1170 & 1462.3 & 30.7 & 169.3 & 1429.1 & 0.977 \\
\hline 18 & 0.20 & 0.1171 & 1466.7 & 31.1 & 168.9 & 1433.3 & 0.977 \\
\hline
\end{tabular}

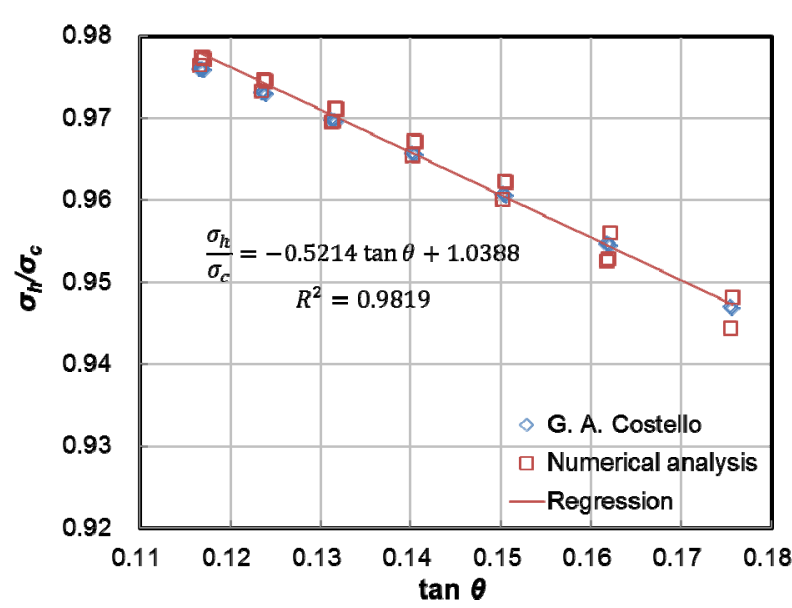

Figure 4. Relation between the helical angle and stress ratio. follows by substituting Equations (1), (2), (3) and (4) into Equation (3).

$$
F=E_{s} \varepsilon_{c}\left[A_{c}+A_{h}\left(-0.5214 \frac{D_{c}+D_{h}}{2 L_{p}}+1.0388\right)\right]
$$

Equation (7) is involves only the strain measured at the center of the core wire and the material and shape parameters of the strand. Following, this equation enables to compute directly the prestress of the strand by simply measuring the strain of the core wire.

\section{Conclusion}

This paper presented a method enabling to compute the prestress of a 7-wire strand simply by measuring the 
strain of the core wire. At the exception of the zones in contact with the helical wires, the stress of the core wire appeared to be nearly constant over the cross section and, except the extremities, the stress of the core wire remained also constant in the longitudinal direction. These observations made it possible to presume that the mean stress of the core wire could be directly obtained from the strain measured at the mid-length center of the core wire. On the other hand, since the stress varied inside the section of the helical wire, the corresponding mean stress was calculated indirectly. In addition, the relation between the mean stresses of the core and helical wires was formulated using the helical angle. Based upon this relation, a formula computing the prestress of the strand directly from the strain measured on the core wire was suggested. The proposed formula in combination with the recently developed method measuring the strain of the core wire can be exploited to determine the prestress of the strand.

\section{Acknowledgements}

This research was supported by a grant from a Strategic Research Project (Development of Smart Prestressing and Monitoring Technologies for Prestressed Concrete Bridges) funded by the Korea Institute of Construction Technology.

\section{REFERENCES}

[1] F. Lanza di Scalea, P. Rizzo and F. Seible, "Stress Measurement and Defect Detection in Steel Strands by Guided Stress Waves," Journal of Materials in Civil Engineering, Vol. 15, No. 3, 2003, pp. 219-227. http://dx.doi.org/10.1061/(ASCE)0899-1561(2003)15:3(2 19)

[2] H.-L. (Roger) Chen and K. Wissawapaisal, "Application of Wigner-Ville Transform to Evaluate Tensile Forces in Seven-Wire Prestressing Strands," Journal of Engineering Mechanics, Vol. 128, No. 11, 2002, pp. 1206-1214. http://dx.doi.org/10.1061/(ASCE)0733-9399(2002)128:11
(1206)

[3] G. Wang, M. L. Wang, Y. Zhao, Y. Chen and B. Sun, "Application of EM Stress Sensors in Large Steel Cables," Smart Structures and Materials 2005: Sensors and Smart Structures Technologies for Civil, Mechanical, and Aerospace Systems, 2005, pp. 395-406.

[4] J. Gao, B. Shi, W. Zhang and H. Zhu, "Monitoring the Stress of the Post-Tensioning Cable Using Fiber Optic Distributed Strain Sensor," Measurement, Vol. 39, No. 5, 2006, pp. 420-428. http://dx.doi.org/10.1016/j.measurement.2005.12.002

[5] J.-M. Kim, H.-W. Kim, Y.-H. Park, I.-H. Yang and Y.-S. Kim, "FBG Sensors Encapsulated into 7-Wire Steel Strand for Tension Monitoring of a Prestressing Tendon," Advances in Structural Engineering, Vol. 15, No. 6, 2012, pp. 907-918.

[6] G. A. Costello, Theory of Wire Rope," 2nd Edition, Springer, New York, 1997, p. 122. http://dx.doi.org/10.1007/978-1-4612-1970-5

[7] S. A. Velinsky, G. L. Anderson and G. A. Costello, "Wire Rope with Complex Cross Sections," Journal of Engineering Mechanics, Vol. 110, No. 3, 1984, pp. 380-391. http://dx.doi.org/10.1061/(ASCE)0733-9399(1984)110:3( 380)

[8] C. Erdönmez and C. Erdem Imrak, "Modeling and Numerical Analysis of the Wire Strand," Journal of Naval Science and Engineering, Vol. 5, No. 1, 2009, pp. 30-38.

[9] G. Shibu, K. V. Mohankumar and S. Devendiran, "Analysis of a Three Layered Straight Wire Rope Strand Using Finite Element Method," Proceedings of the World Congress on Engineering 2011, 2011, Vol. 3.

[10] I. Gerdemeli, S. Kurt and A. S. Anil, "Analysis with Finite Element Method of Wire Rope," Vol. 78, No. 1, 2013. http://mech-ing.com/journal/Archive/2012/11/185_Kurt.p df

[11] Korean Standards, "Uncoated Stress-Relieved Steel Wires and Strands for Prestressed Concrete," KSD 7002, 2011.

[12] W. G. Jiang and J. L. Henshall, "The Analysis of Termination Effects in Wire Strand Using the Finite Element Method," The Journal of Strain Analysis for Engineering Design, Vol. 34, No. 1, 1999, pp. 31-38. http://dx.doi.org/10.1243/0309324991513605 